\title{
A Novel High Resolution Spectrum Sensing Algorithm for Cognitive Radio Applications
}

\author{
Bommidi Sridhar, Dr T.Srinivasulu \\ Associate Professor, Guru Nanak Institutions Technical Campus Hyderabad, 501506, India. \\ Professor, Ku College Of Engineering\& Technology, Kakatiya University Warangal, 506009, India
}

\begin{abstract}
The spectrum sensing related issues has come up with new aspects with cognitive radio and opportunistic spectrum access concepts. Spectrum sensing by far is the most important component for the establishment of cognitive radio. In this paper, some of the spectrum sensing methodologies for cognitive radio is presented. The Challenges associated with spectrum sensing are given and enabling spectrum sensing methods are reviewed. In this paper, an efficient recursive least square (ERLS) algorithm is proposed for improving the power signal spectral estimation. The proposed ERLS algorithm is the combination of wavelet algorithm and artificial neural network (ANN).

Key words : Cognitive radio, dynamic spectrum access, multi-dimensional spectrum sensing, WSS-wide sense stationary, CSD-cyclic spectral density, FFT-fast fourier transforms, ERLS-effective recursive least square algorithm, ANN-artificial neural networks.
\end{abstract}

\section{Introduction}

The requirement of a higher data rates is increasing as a result of the transition from voice-only communications to modern multimedia type applications. Given the limitations of the natural frequency spectrum, it becomes obvious that the current static frequency allocation schemes are insufficient to accommodate the requirements of an increasing number of higher data rate devices. As a result, innovative techniques that can offer new ways of exploiting the available spectrum are needed. Cognitive radio arises to be a tempting solution to the spectral congestion problem by introducing opportunistic usage of the frequency bands that are not heavily occupied by licensed users [1], [2]. In this paper, we use the definition adopted by Federal Communications Commission (FCC): "Cognitive radio: A radio or system that senses its operational electromagnetic environment and can dynamically and autonomously adjust its radio operating parameters to modify system operation, such as maximize throughput, mitigate interference, facilitate interoperability, access secondary markets." [2]. Hence, one main aspect of cognitive radio is related to autonomously exploiting locally unused spectrum to provide new paths to spectrum access . In cognitive radio terminology, primary users can be defined as the users who have higher priority or legacy rights on the usage of a specific part of the spectrum. On the other hand, secondary users, which have lower priority, exploit this spectrum in such a way that they do not cause interference to primary users. Therefore, secondary users need to have cognitive radio capabilities, such as sensing the spectrum reliably to check whether it is being used by a primary user and to change the radio parameters to exploit the unused part of the spectrum. Being the focus of this paper, spectrum sensing by far is the most important component for the establishment of cognitive radio. Spectrum sensing is the task of obtaining awareness about the spectrum usage and existence of primary users in a geographical area. This awareness can be obtained by using geolocation and database, by using beacons, or by local spectrum sensing at cognitive radios [3]. Although spectrum sensing is traditionally understood as measuring the spectral content, or measuring the radio frequency energy over the spectrum; when cognitive radio is considered, it is a more general term that involves obtaining the spectrum usage characteristics across multiple dimensions such as time, space, frequency, and code. It also involves determining what types of signals are occupying the spectrum including the modulation, waveform, bandwidth, carrier frequency, etc.. However, this requires more powerful signal analysis techniques with additional computational complexity.

\section{Multi-Dimensional Spectrum Awareness}

The definition of opportunitydetermines the ways of measuring and exploiting the spectrum space. The conventional definition of the spectrum opportunity, which is often defined as "a band of frequencies that are not being used by the primary user of that band at a particular time in a particular geographic area" [4], only exploits three dimensions of the spectrum space: frequency, time, and space. Conventional sensing methods usually relate to sensing the spectrum in these three dimensions. However, there are other dimensions that need to be explored further for spectrum opportunity. For example, the code dimension of the spectrum space has not been short. The radio space with the introduced dimensions can be defined as "a theoretical hyperspace occupied by radio signals, which has dimensions of location, angle of arrival, frequency, time, and possibly 
others" [5]. This hyperspace is called electrospace, transmission hyperspace, radio spectrum space, or simply spectrum space by various authors, and it can be used to describe how the radio environment can be shared among multiple (primary and/or secondary) systems [6]. Spectrum sensing should include the process of identifying occupancy in all dimensions of the spectrum space and finding spectrum holes, or more precisely spectrum space holes. For example a certain frequency can be occupied for a given time, but it might be empty in another time. Hence, temporal dimension is as important as frequency dimension.

\section{Challenges}

Before getting into the details of spectrum sensing techniques, challenges associated with the spectrum sensing for cognitive radio are given in this section.
A. Hardware Requirements
B. Hidden Primary User Problem
C. Detecting Spread Spectrum Primary Users
D. Sensing Duration and Frequency
E. Decision Fusion in Cooperative Sensing
F. Security

\section{METHODS FORCOGNITIVE RADIO}

\section{Spectrum Sensing}

The present literature for spectrum sensing is still in its early stages of development. A number of different methods are proposed for identifying the presence of signal transmissions. In some approaches, characteristics of the identified transmission are detected for deciding the signal transmission as well as identifying the signal type. In this section, some of the most common spectrum sensing techniques in the cognitive radio literature are explained.

\section{A. Energy Detector Based Sensing}

Energy detector based approach, also known as radiometry or periodogram, is the most common way of spectrum sensing because of its low computational and implementation complexities [7], it is more generic (as compared to methods given in this section) as receivers do not need any knowledge on the primary users' signal. The signal is detected by comparing the output of the energy detector with a threshold which depends on the noise floor [14]. Let us assume that the received signal has the following simple form $y(n)=s(n)+w(n)$, (1) where $\mathrm{s}(\mathrm{n})$ is the signal to be detected, $\mathrm{w}(\mathrm{n})$ is the additive white Gaussian noise (AWGN) sample, and $\mathrm{n}$ is the sample index. Note that $\mathrm{s}(\mathrm{n})=0$ when there is no transmission by primary user. The decision metric for the energy detector can be written as

$M=\sum_{n=0}^{N}|y(n)|^{2}, \quad$ (2) Where $\mathrm{N}$ is the size of the observation vector. The decision on the occupancy of a band can be obtained by comparing the decision metric $M$ against a fixed threshold $\lambda$. This is equivalent to distinguishing between the following two hypotheses: $\mathrm{HO}: \mathrm{y}(\mathrm{n})=\mathrm{w}(\mathrm{n}),(3)$,

H1: $y(n)=s(n)+w(n)$ (4) The performance of the detection algorithm can be summarized with two probabilities: probability of detection PD and probability of false alarm PF. PD is the probability of detecting a signal on the considered frequency when it truly is present. Thus, a large detection probability is desired. It can be formulated as $\quad P D=\operatorname{Pr}(M>\lambda \mid H 1)$. (5) $P F$ is the probability that the test incorrectly decides that the considered frequency is occupied when it actually is not, and it can be written as $\mathrm{PF}=\operatorname{Pr}(\mathrm{M}>\lambda \mid \mathrm{H} 0)$. (6) $\mathrm{PF}$ should be kept as small as possible in order to prevent underutilization of transmission opportunities. The decision threshold $\lambda$ can be selected for finding an optimum balance Between PD and PF.. In practice, the threshold is chosen to obtain a certain false alarm rate [15]. Hence, knowledge of noise variance is sufficient for selection of a threshold. The white noise can be modeled as a zero-mean Gaussian random variable with variance, i.e. $\mathrm{w}(\mathrm{n})=№\left(0, \sigma_{w}^{2}\right)$. For a simplified analysis, let us model the signal term as a zero-mean Gaussian variable as well,i.e. $\mathrm{s}(\mathrm{n})=№\left(0, \sigma_{s}^{2}\right)$..The model for $\mathrm{s}(\mathrm{n})$ is more complicated as fading should also be considered. Because of these assumptions, the decision metric (2) follows chi-square distribution with $2 \mathrm{~N}$ degrees of freedom $\chi_{2 N}^{2}$ and hence, it can be modeled as

$M=\left\{\begin{array}{c}\left(\frac{\sigma_{W}^{2}}{2}\right) \chi_{2 N}^{2}, H 0, \\ \left(\frac{\sigma_{W}^{2}+\sigma_{S}^{2}}{2}\right) \chi_{2 N}^{2}, H 1\end{array}\right.$ 


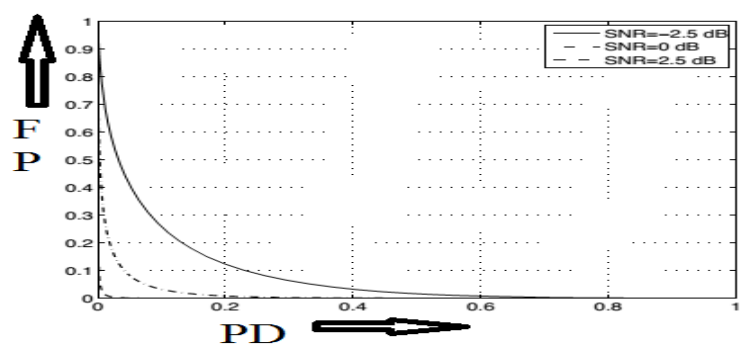

Fig-01: ROC curves for energy detector based spectrum sensing under different SNR values.

For energy detector, the probabilities PF and PD can be calculated as [41]

$\mathrm{PF}=1-\Gamma\left(\mathrm{L}_{\mathrm{f}} \mathrm{L}_{\mathrm{t}}, \lambda / \sigma_{w}^{2}\right)$,

$\mathrm{PD}=1-\Gamma\left(\mathrm{L}_{\mathrm{f}} \mathrm{L}_{\mathrm{t}}, \lambda\left(\sigma_{w}^{2}+\sigma_{s}^{2}\right)\right),(9)$

Where $\lambda$ is the decision threshold, and $\Gamma(\mathrm{a}, \mathrm{x})$ is the incomplete gamma function as given in [16] (ref. Equation 6.5.1). In order to compare the performances for different threshold values, receiver operating characteristic (ROC) curves can be used. ROC curves allow us to explore the relationship between the sensitivity (probability of detection) and specificity (false alarm rate) of a sensing method for a variety of different thresholds, thus allowing the determination of an optimal threshold. Fig. 01 shows the ROC curves for different SNR values. SNR is defined as the ratio of the primary user's signal power to noise power, i.e. $\mathrm{SNR}=\sigma_{s}^{2} / \sigma_{w}^{2}$. The number of used samples is set to15 in this figure, i.e. $\mathrm{N}=15$ in (2). As this figure clearly shows, the performance of the threshold detector increases at high SNR values.

\section{B. Waveform-Based Sensing}

Known patterns are usually utilized in wireless systems to assist synchronization or for other purposes. Such patterns include preambles, mid ambles, regularly transmitted pilot patterns, spreading sequences etc. A preamble is a known sequence transmitted before each burst and a mid amble is transmitted in the middle of a burst or slot. In the presence of a known pattern, sensing can be performed by correlating the received signal with a known copy of itself [11]. This method is only applicable to systems with known signal patterns, and it is termed as waveform-based sensing or coherent sensing. In [11], it is shown that wave form based sensing outperforms energy detector based sensing in reliability and convergence time. Furthermore, it is shown that the performance of the sensing algorithm increases as the length of the known signal pattern increases. Using the same model given in (1), the waveform-based sensing metric can be obtained as [11]

$M=\operatorname{Re}\left[\sum_{n=1}^{N} y(n) s^{*}(n)\right]$

Where $*$ represents the conjugation operation. In the absence of the primary user, the metric value becomes

$M=\operatorname{Re}\left[\sum_{n=1}^{N} w(n) s^{*}(n)\right] \quad$,(11) In this paper, time-domain sampling is explained as an example. Modified versions of the method explained in this paper can be used in frequency domain as well. Likewise, the method given in this paper can be modified depending on the available pattern. Similarly, in the presence of a primary user's signal, the sensing metric becomes

$M=\sum_{n=1}^{N}|s(n)|^{2}+\operatorname{Re}\left[\sum_{n=1}^{N} w(n) s^{*}(n)\right],(12)$

The decision on the presence of a primary user signal can be made by comparing the decision metric $\mathrm{M}$ against a fixed threshold $\lambda \mathrm{w}$. For analyzing the WLAN channel usage characteristics, packet preambles of IEEE 802.11b [17] signals are exploited in [12]. Measurement results presented in [09] show that waveform-based sensing requires short measurement time; however, it is susceptible to synchronization errors. Uplink packet preambles are exploited for detecting Worldwide Interoperability for Microwave Access (WiMAX) signals in [13].

\section{Cyclostationarity-Based Sensing}

Cyclostationarity feature detection is a method for detecting primary user transmissions by exploiting the cyclostationarity features of the received signals [07]. Cyclostationary features are caused by the periodicity in the signal or in its statistics like mean and autocorrelation [21] or they can be intentionally induced to assist spectrum sensing [22]. Instead of power spectral density (PSD), cyclic correlation function is used for detecting signals present in a given spectrum. The cyclostationarity based detection algorithms can differentiate noise from primary users' signals. This is a result of the fact that noise is wide-sense stationary (WSS) with no correlation while modulated signals are cyclostationary with spectral correlation due to the redundancy of signal periodicities [19]. Furthermore, cyclostationarity can be used for distinguishing among different types of transmissions and primary users [21]. The cyclic spectral density (CSD) function of a received signal (1) can be calculated as [80]

$$
\begin{aligned}
& S(f, \alpha)=\sum_{\tau=-\infty}^{\infty} R_{y}^{\alpha}(\tau) e^{-j 2 \pi f \tau} \text { (13) Where } \\
& R_{y}^{\alpha}(\tau)=E\left[y(n+\tau) y^{*}(n-\tau)\right] e^{j 2 \pi \alpha n}
\end{aligned}
$$


is the cyclic autocorrelation function (CAF) and $\alpha$ is the cyclic frequency. The CSD function outputs peak values when the cyclic frequency is equal to the fundamental frequencies of transmitted signal $\mathrm{x}(\mathrm{n})$. Cyclic frequencies can be assumed to be known [18] or they can be extracted and used as features for identifying transmitted signals [20]. The OFDM waveform is altered before transmission in [22] in order to generate system specific signatures or cycle-frequencies at certain frequencies. These signatures are then used to provide an effective signal classification mechanism.

\section{Matched-Filtering}

Matched-filtering is known as the optimum method for detection of primary users when the transmitted signal is known [23]. The main advantage of matched filtering is the short time to achieve a certain probability of false alarm or probability of miss detection [24] as compared to other methods that are discussed in this section. In fact, the required number of samples grows as $\mathrm{O}(1 / \mathrm{SNR})$ for a target probability of false alarm at low SNRs for matched filtering [24]. However, matched-filtering requires cognitive radio to demodulate received signals. Hence, it requires perfect knowledge of the primary users signaling features such as bandwidth, operating frequency, modulation type and order, pulse shaping, and frame format. Moreover, since cognitive radio needs receivers for all signal types, the implementation complexity of sensing unit is impractically large [10]. Another disadvantage of match filtering is large power consumption as various receiver algorithms need to be executed for detection.

\section{E. Wavelets in Sensing Methods}

Other alternative spectrum sensing methods include wavelet transform based estimation.

In [25], wavelets are used for detecting edges in the PSD of a wideband channel. Once the edges, which correspond to transitions from an occupied band to an empty band or vice versa, are detected, the powers within bands between two edges are estimated. Using this information and edge positions, the frequency spectrum can be characterized as occupied or empty in a binary fashion. The assumptions made in [25], however, need to be relaxed for building a practical sensing algorithm. The method proposed in [25] is extended in [26] by using sub-Nyquist sampling. Assuming that the signal spectrum is sparse, sub-Nyquist sampling is used to obtain a coarse spectrum knowledge in an efficient way. Analog implementation of wavelet-transform based sensing is proposed in [08] for coarse sensing. Analog implementation yields low power consumption and enables real time operation. Multi-resolution spectrum sensing is achieved by changing the basis functions without any modification to sensing circuitry in [08]. Basis function is changed by adjusting the wavelet's pulse width and carrier frequency. Hence, fast sensing is possible by focusing on the frequencies with active transmissions after an initial rough scanning.

\section{Proposed Novel Sensing Algorithm (Erlf)}

In RLS algorithm based power spectral estimation, the frequency variation of the signal is determined by Fast Fourier Transform (FFT). The FFT based signal extraction is more costly because, the estimation is based on the bandwidth variation of the signal. But, the wavelet algorithm based feature extraction is simple than the FFT. Also, the error variation is analyzed on iteration based, so the complexity is increased. The artificial intelligence technique predicts the error variation at different time. So, the spectral estimation is very simple than the RLS algorithm. In this paper, an ERLS algorithm based spectral estimation technique is proposed. The proposed algorithm is based on the wavelet and neural network. Some drawbacks of RLS algorithm is it requires high computational power and the output obtained is numerically instability. So, the spectral efficiency of the signal is affected and a power error occurs in the estimator. To analyze the estimation performance of RLS algorithm, the capon and APES based spectral analysis methods are used. In the adaptive RLS algorithm based spectral estimator, the power error of the signal is predicted by forward and backward predictors. Both these forward and backward predictors can able to predict only the linear signal power error and it can't predict the non-linearity signal power error. Then, the frequency domain variables of the signal are computed by Fast Fourier Transform (FFT). The main limitation of the frequency and bandwidth of FFT spectrum analyzers is the analogue to digital converter (ADC). So, the high level of performance required by the $\mathrm{ADC}$ means that this item is a very high cost item. In addition to all the other processing and display circuitry required, this results in the costs rising for these items. To overcome these problems, in this paper an efficient recursive least square (ERLS) algorithm is proposed for improving the power signal spectral estimation. The spectral estimation capability of RLS algorithm is enhanced by ANN and wavelet transform. The proposed ANN is one of the artificial intelligence (AI) techniques, used to predict the power error of linear and non-linear signals. So, the spectral efficiency and stability of the time varying signal is improved. Then, the frequency variation of the signal is computed by wavelet transform. Because, the wavelet transform provide the frequency of the signals as well as the time related to time varying signal. So, the limitation of the FFT spectrum analyzer 
is reduced and the cost effective problem is minimized. Also, the computational power of the proposed algorithm is reduced

\section{A. Proposed ERLS Algorithm Based Spectral Estimation}

In the proposed ERLS algorithm, the frequency components of the signal are extracted by wavelet transform and the signal error is determined by AI technique. The spectral estimation signals desired responses are $\{d(1), d(2), \ldots \ldots \ldots, d(N)\}$ and the input samples of the desired responses are $\{s(1), s(2), \ldots \ldots \ldots, s(N)\}$. Then, the linear filter output of the desired response is described as,

$$
x(n)=\sum_{k=0}^{M} w_{k} s(n-k), \quad n=0,1,2, \ldots \ldots . . .
$$

Then, the weight is applied in the filter output recursively and the error of the response is reduced. The recursive weight parameters are $\left\{w_{0}(1), w_{1}(2), \ldots \ldots \ldots, w(n)\right\}$ and the sum of minimum square error is given as,

$\varepsilon(n)=\varepsilon\left(w_{0}(n), w_{1}(n), \ldots . ., w_{M-1}(n)\right)$

i.e., $\varepsilon(n)=\sum_{i=i_{1}}^{n} \rho(n, i)\left[e(i)^{2}\right] \quad 0<\rho(n, i) \leq 1$

where, $\rho(n, i)=\lambda^{n-i}, e(i)$ is the error value of the signal and $i=1,2,3 \ldots \ldots, n$. The formula for determining the signal error is given as,

$e(i)=d(i)-x(i)$

Then, the error signals of the desired response are applied to the minimum square error function and a new value is obtained i.e., in equation (17). The new minimum square error function is,

$\varepsilon(n)_{\text {new }}=\sum_{i=i_{1}}^{n} \lambda^{n-i}\left[d(i)-\sum_{k=0}^{M-1} w_{k}(n) s(i-k)\right]^{2}$

Similarly, the minimum least square error of the desired signal is achieved. This process is performed iteratively and finally, the output of the least square algorithm is determined by the following formula,

$$
L(n)=\frac{1}{\lambda}\left(L(n-1)-L^{\prime}\right)
$$

where, $L(n)$ is the recursive variables, $L^{\prime}$ is the recursive value of the estimator i.e., $L^{\prime}=k(n) \psi$, $k(n)=\frac{\psi^{T}}{\gamma}, \gamma=\lambda+\psi s$ and $\psi=s^{T}(n) L(n-1)$. The recursive process complexity is reduced by using neural network and wavelet transform. In eq. (18), the error signal value $e(i)$ of corresponding input samples are determined by using neural network and the frequencies of input samples are extracted by wavelet transform.

\section{B. Wavelet and ANN Based Adaptive Spectral Estimation}

Extraction of Frequency Components by Wavelet Transform: The wavelet transform is a mathematical function, used to divide a given signal into different scale components. Here, the purpose of wavelet transform is to determine the samples disturbance. The input samples of the desired frequency $\{s(1), s(2), \ldots \ldots \ldots, s(N)\}$ are applied to the input of wavelet transform, and the frequency variation of the samples is determined. The input samples are convoluted with the wavelet signal $\omega(t)$ and the approximate frequency coefficient is determined. The approximate frequency coefficient function $\left(C_{m, n}\right)$ is illustrated as,

$$
C_{m . n}=\int_{-\infty}^{\infty} s(t) \phi_{m, n}(t) d t
$$

where, $m$ is the scale and $n$ is the location of the signal approximation coefficient. The finite length of the input sample is $N$. So, the range of the scales can be investigated as $0<m<M$. Hence, the approximation of the signal can be described as, 
$S_{0}(t)=S_{M}(t)+\sum_{m=1}^{M} d_{m}(t)$

where, the mean signal approximation at scale $M$ is $x_{M, n} \phi_{M, n}(t)$ and the digital signal approximation corresponding to scale $m$ for finite length signal is given in eqn.(23). Then, the signal approximation at specific scale is the combination of the lower scale signal and it is described in eqn. (24).

$$
\begin{aligned}
d_{m}(t) & =\sum_{n=0}^{M-m} C_{m, n} \phi_{m, n}(t) \\
x_{m}(t) & =s_{m-1}(t)-d_{m}(t)
\end{aligned}
$$

The output of the wavelet transform is based on the input samples of the desired response. Then, the error of the input samples is determined by using the eq. (18). The network process is explained in the following section.

Determining Signal Error by Artificial Neural Network (ANN): The ANN plays an important role in signal processing, used for analyzing the signal complexity or error [28]. Here, the purpose of artificial intelligence technique is to determine the error signal $e(i)$ for the corresponding $d(i)$ and $x(i)$ values. In proposed spectral estimation, the feed forward type $\mathrm{NN}$ is used, which consists of three layers namely, input layer, hidden layer, and output layer. Here, the inputs to the neural network are denoted as $d(i)$ and $x(i)$ respectively, and the output is error signal $e(i)$. Once the process gets completed, the network is well-trained and it would be suitable for providing $e(i)^{N N}$ values for any $d(i)$ and $x(i)$. Then, the performance of the proposed ERLS algorithm is evaluated with two spectral estimators.

\section{Performance Estimation of Proposed ERLS algorithm}

The performance of the proposed algorithm is evaluated with two spectral estimators. Here, the amplitude and phase estimation (APES) and capon estimators are used for analyzing the performance of the proposed ERLS algorithm. The formula used for computing the spectral estimation of the proposed algorithm is described below. The APES based spectral estimator is given as,

$$
\begin{gathered}
\alpha_{A P E S}\left(\omega_{k}\right)=\frac{a_{M}^{H}\left(\hat{Q}-Y_{\omega} Y_{\omega}^{H}\right)^{-1} Y_{\omega}}{a_{M}^{H}\left(\hat{Q}-Y_{\omega} Y_{\omega}^{H}\right)^{-1} a_{\omega}} \\
\text { i.e. } \alpha_{A P E S}\left(\omega_{k}\right)=\frac{\lambda_{\omega}}{\psi_{\omega}-\psi_{\omega} \gamma_{\omega}+\left|\lambda_{\omega}\right|^{2}}
\end{gathered}
$$

where, $\gamma_{\omega}=Y_{\omega}^{H} \hat{Q}^{-1} Y_{\omega}$

The capon based spectral estimator is given as,

$$
\alpha_{\text {Capon }}\left(\omega_{k}\right)=\arg \max _{\omega_{k}}\left|\frac{\lambda_{\omega}}{\psi_{\omega}}\right|
$$

where, $\lambda_{\omega}=a_{M}^{H} \hat{Q}^{-1} Y_{\omega}$

$$
\psi_{\omega}=a_{M}^{H} \hat{Q}^{-1} a_{\omega}
$$

These two spectral estimators are used to estimate the spectral lines of the processing signal. Then, based on the output of the estimators, the efficiency of the proposed algorithm is analyzed.

\section{Results and Discussion}

The proposed ERLS algorithm is implemented in the working platform of MATLAB version 7.12. Here, the output of the proposed algorithm is compared with the existing RLS algorithm [27]. The estimation efficiency of the proposed algorithm is analyzed by means of APES and Capon methods. Then, the filter weight and the estimated weights of the estimator are compared. The estimated weight convergences at different samples are analyzed. Then, the adaptive-ness of the proposed algorithm is analyzed at different samples, error values, and corresponding weight values. The system output processing signal is shown in Fig.02. Then from the system output, 2000 samples are chosen for analyzing the error present in the signal. The performance of 
system output error for the RLS algorithm is shown in Fig.03. The performance of ERLS algorithm based error is illustrated in Fig.04.

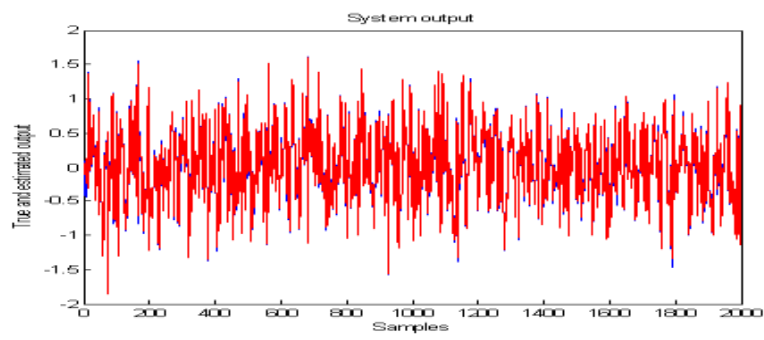

Fig 02: Performance of the System Output Signal.

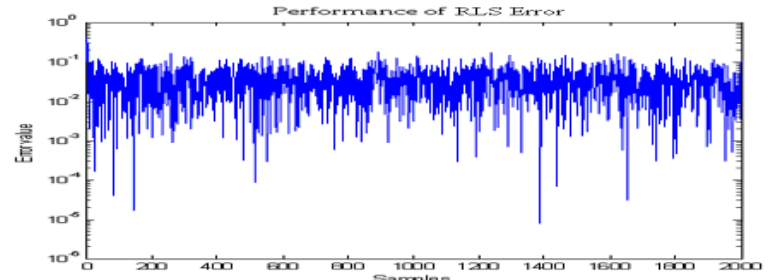

Fig 03: Performance of RLS Algorithm Error vs. Samples.

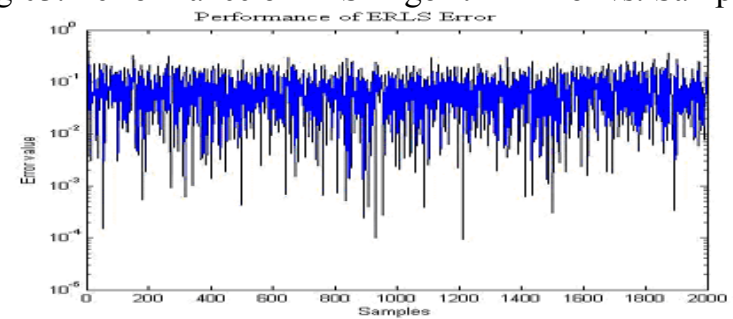

Fig 04: Performance of ERLS Algorithm Error vs. Samples.

Then, the spectral estimation performance of the proposed ERLS algorithm is analyzed with the APES and Capon based spectral estimator. The estimators are performed based on the filter bank approach. In the filter bank approach based estimation, the spectral error lines are estimated by the measured signal from the filter. Then, the network performances are plotted. The time samples are represented in frequency and the estimated error is denoted as amplitude. The neural network performance in Regression, validation, and training state are illustrated in Fig.06.

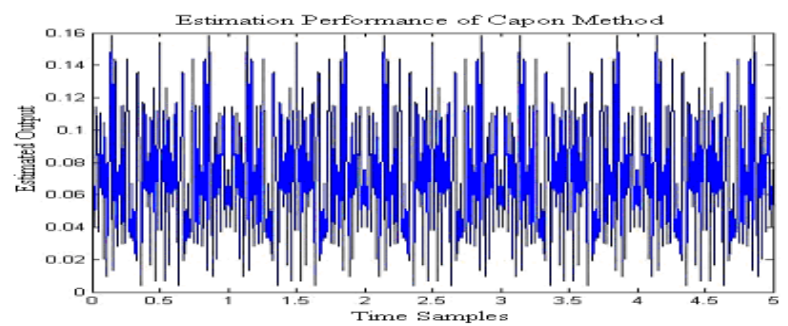

Fig 05: Performance of Spectral Error Estimation in Capon Method.

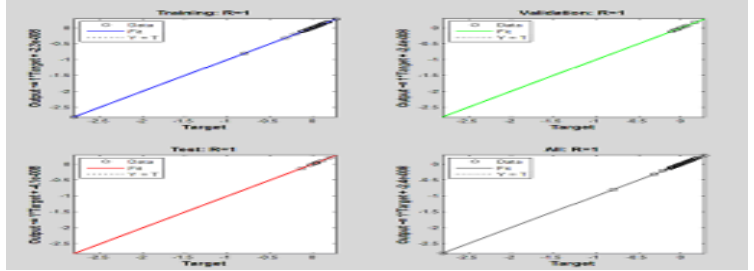

(i)

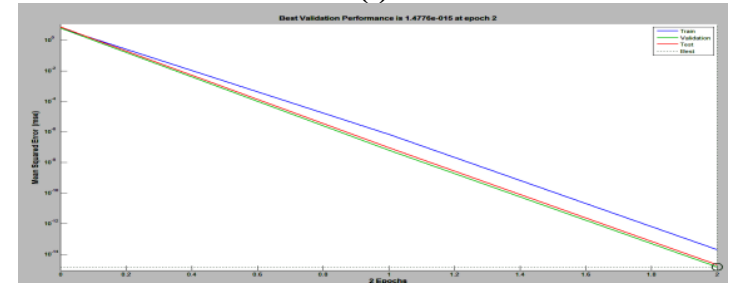

(ii) 


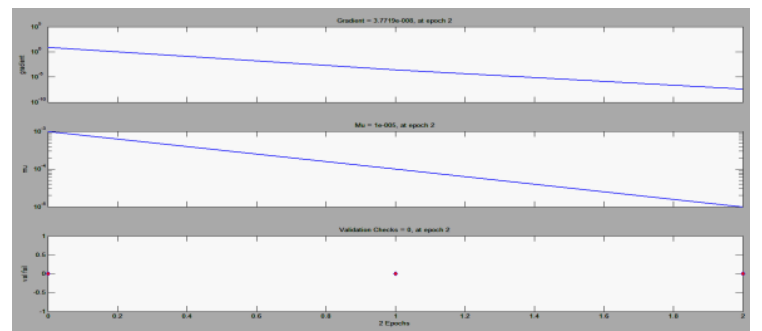

(iii)

Fig 06: The network Performance Plots (i) Regression Analysis, (ii) Network Validation performance, and (iii) Training State.

Then, the adaptiveness of the proposed ERLS algorithm is analyzed. The adaptive-ness is analyzed based on the samples, frequency, and spectral estimated error values. The performance of the adaptive spectral estimation is shown in Fig.07. From the adaptive performance, the spectral estimation efficiency of the proposed method is revealed. The proposed ERLS algorithm based spectral line error estimation of the signal is accurate. In RLS based estimation, the frequency domain is adapted without stabilization and so, it results in severe error magnification. But, the proposed efficient recursive least square (ERLS) algorithm has less computational complexity and it has achieved better stabilization.

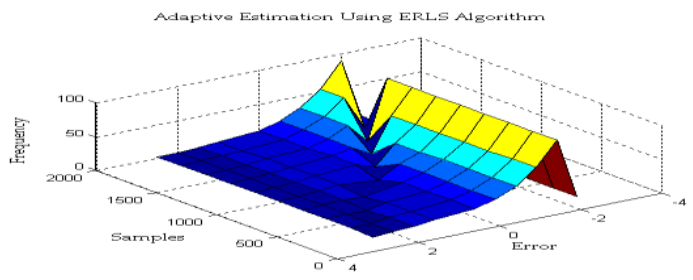

Fig 07: Performance of Adaptive Estimation by ERLS Algorithm.

\section{Conclusions}

The proposed ERLS algorithm was implemented in MATLAB version 7.12 and the spectral estimation performances were evaluated. The performance evaluation of the spectral estimation was based on the system output signal. The system output error of the proposed ERLS algorithm and the existing RLS algorithm was compared. From the comparison, it was found that the error estimation sensitivity of RLS was less compared to ERLS algorithm. Then, the estimated weights and the weight convergence time of both algorithms were analyzed. The analyzed results have revealed that the proposed algorithm have fast convergence rate for estimating the error at different samples. Then, the performance of the ERLS algorithm was analyzed with two performance measuring methods. The APES and Capon methods were measured the error at different time samples. The estimator output was more accurate, so the estimation efficiency of the proposed algorithm was improved. Finally, the adptiveness of the proposed algorithm was analyzed. The adaptiveness of the proposed algorithm was based on the samples, frequency, and estimated error from the system output. Overall, the proposed ERLS algorithm based spectral estimation has given better estimation results than the existing RLS algorithm.

\section{References}

[1] I. Mitola, J. and J. Maguire, G. Q.,“Cognitive radio: making software radios more personal,” IEEE Personal Commun. Mag., vol. 6, no. 4 pp. 13-18, Aug. 1999.

[2] Federal Communications Commission, "Notice of proposed rule making and order: Facilitating opportunities for flexible, efficient, and reliable spectrum use employing cognitive radio technologies," ET Docket No. 03-108, Feb. 2005.

[3] Federal Communications Commission, "Notice of proposed rule making: Unlicensed operation in the TV broadcast bands," ET Docket No. 04-186 (FCC 04-113), May 2004.

[4] P. Kolodzy et al., "Next generation communications: Kickoff meeting," in Proc. DARPA, Oct. 2001.

[5] R. Matheson, "The electrospace model as a frequency management tool," in Int. Symposium On Advanced Radio Technologies, Boulder,

[6] A.L.Drozd,I.P.Kasperovich ,C.E.Carroll and A.C.Black burn, "Computational electromagnetics applied to analyzing the efficient utilization of the RF transmission hyperspace," inProc. IEEE/ACES

[7] S. Shankar, C. Cordeiro, and K. Challapali, "Spectrum agile radios: utilization and sensing architectures," inProc. IEEE Int. Symposium on New Frontiers in Dynamic Spectrum Access Networks, Baltimore,

[8] Y. Hur, J. Park, W. Woo, K. Lim, C. Lee, H. Kim, and J. Laskar, “A wideband analog multi-resolution spectrum sensing (MRSS) technique for cognitive radio (CR) systems," in Proc. IEEE Int. Symp. Circuits and Systems, Island of Kos, Greece, May 2006, pp. 4090-4093

[9] D. Cabric, A. Tkachenko, and R. Brodersen, "Spectrum sensing measurements of pilot, energy, and collaborative detection," inProc. IEEE Military Commun. Conf., Washington, D.C., USA, Oct. 2006, 
[10] D. Cabric, S. Mishra, and R. Brodersen, "Implementation issues in spectrum sensing for cognitive radios," in Proc. Asilomar Conf. on Signals, Systems and Computers,vol.1,PacificGrove,California, USA, Nov. 2004, pp. 772-776.

[11] H. Tang, "Some physical layer issues of wide-band cognitive radio systems," inProc. IEEE Int. Symposium on New Frontiers in Dynamic Spectrum Access Networks, Baltimore, Maryland, USA, Nov. 2005, pp.

[12] S. Geirhofer, L. Tong, and B. Sadler, "A measurement-based model for dynamic spectrum access in WLAN channels," inProc. IEEE Military Commun. Conf., Washington, D.C., USA, Oct. 2006.

[13] S. t. B. S. M. Mishra, R. Mahadevappa, and R. W. Brodersen, "Cognitive technology for ultra-wideband/WiMax coexistence," in Proc. IEEE Int. Symposium on New Frontiers in Dynamic Spectrum Access Networks, Dublin, Ireland, Apr. 2007,

[14] H. Urkowitz, "Energy detection of unknown deterministic signals," Proc. IEEE, vol. 55, pp. 523-531,

[15] J. Lehtom" aki, M. Juntti, H. Saarnisaari, and S. Koivu, "Threshold setting strategies for a quantized total power radiometer,"IEEE Signal Processing Lett., vol. [16] M. Abramowitz and I. Stegun, Eds., Handbook of Mathematical Functions With Formulas, Graphs, and Mathematical Tables,9thed. National Bureau of Standards, 1970.

[17] Supplement to IEEE standard for information technology telecommunications and information exchange between systems - local and metropolitan area networks - specific requirements. Part 11: wireless LAN Medium Access Control (MAC) and Physical Layer (PHY) specifications: high-speed physical layer externsion in the $2.4 \mathrm{GHz}$ band, The Institute of Electrical and Electronics Engineering, Inc. Std. IEEE 802.11b, Sept. 1999.

[18] M. Oner and F. Jondral, "Cyclostationarity based air interface recognition for software radio systems," inProc. IEEE Radio and Wireless Conf., Atlanta, Georgia, USA, Sept. 2004, pp. 263-266.

[19] D. Cabric and R. W. Brodersen, "Physical layer design issues unique to cognitive radio systems," inProc. IEEE Int. Symposium on Personal, Indoor and Mobile Radio Commun.,vol.2,Berlin,Germany,Sept. 2005, pp. 759-763.

[20] A. Fehske, J. Gaeddert, and J. Reed, "A new approach to signal classification using spectral correlation and neural networks," inProc. IEEE Int. Symposium on New Frontiers in Dynamic Spectrum Access Networks, Baltimore, Maryland, USA, Nov. 2005, pp. 144-150. WRAN systems," inProc. IEEE Int. Conf. Advanced Communication Technology, vol. 3, Feb. 2006.

[21] J. Lund' en, V. Koivunen, A. Huttunen, and H. V. Poor, "Spectrum sensing in cognitive radios based on multiple cyclic frequencies," in Proc. IEEE Int. Conf. Cognitive Radio Oriented Wireless Networks and Commun. (Crowncom), Orlando, Florida, USA, July/Aug. 2007.

[21] U. Gardner, WA, "Exploitation of spectral redundancy in cyclostationary signals,"IEEE Signal Processing Mag., vol. 8, no. 2, pp. 14-36, 1991.

[22] K. Maeda, A. Benjebbour, T. Asai, T. Furuno, and T. Ohya, "Recognition among OFDM-based systems utilizing cyclostationarityinducing transmission," in Proc. IEEE Int. Symposium on New Frontiers in Dynamic Spectrum Access Networks, Dublin, Ireland, Apr. 2007, pp. 516-523.

[23] J. G. Proakis, Digital Communications, 4th ed. McGraw-Hill, 2001. [92] R. Tandra and A. Sahai, "Fundamental limits on detection in low SNR under noise uncertainty," inProc. IEEE Int. Conf. Wireless Networks, Commun. and Mobile Computing, vol. 1, Maui, HI, June 2005, pp. 464-469.

[24] R. Tandra and A. Sahai, "Fundamental limits on detection in low SNR under noise uncertainty," inProc. IEEE Int. Conf. Wireless Networks, Commun. and Mobile Computing, vol. 1, Maui, HI, June 2005, pp. 464-469.

[25] Z. Tian and G. B. Giannakis, "A wavelet approach to wideband spectrum sensing for cognitive radios," inProc. IEEE Int. Conf. Cognitive Radio Oriented Wireless Networks and Commun. (Crowncom), Mykonos Island, Greece, June 2006.

[26] Z. Tian and G. Giannakis, "Compressed sensing for wideband cognitive radios," in Proc. IEEE Int. Conf. on Acoustics, Speech, and Signal Processing, vol. 4, Honolulu, Hawaii, USA, Apr. 2007, pp. 1357-1360.

[27] George Othon Glentis, "Efficient Algorithms for Adaptive Capon and APES Spectral Estimation", IEEE Transactions on Signal Processing, Vol.58, No.1, pp.84-96, January 2010

[28] Anupama Pande, Ashok Kumar Thakur and Swapnoneel Roy, "Complex-Valued Neural Network in Signal Processing: A Study on the Effectiveness of Complex Valued Generalized Mean Neuron Model", International Journal of Computer Science and Engineering, Vol.2, No.3, pp.129-134, 2008 